\title{
Inibição Enzimática, Neuroproteção e Tratamento da Doença de Parkinson
}

\author{
Luiz Augusto Franco de Andrade* \& \\ Henrique Ballalai Ferraz**
}

\section{RESUMO}

Os autores apresentam uma revisāo dos mecanismos bioquimicos envolvidos no metabolismo da dopamina no cérebro, dos sistemas enzimáticos envolvidos na metabolização e catabolização deste neurotransmissor e das drogas que podem interferir neste metabolismo. $O$ conceito de neuroproteção cerebral no tratamento da doença de Parkinson também é revisto. O papel de inibidores da MAO, como a moclobemida e a selegilina, já existentes no mercado, assim como de novas drogas ainda nào comercializadas ou em vias de o serem, como a lazabemida e o inibidor da COMT tolcapone, sāo discutidos em sua utilidade no tratamento sintomático ou neuroprotetor da doença de Parkinson.

\section{UNITERMOS}

Inibidores da MAO, inibidores da COMT, neuroproteção, doença de Parkinson.

\section{INTRODUÇÃO}

A dopamina, a noradrenalina, a serotonina, a adrenalina, a tiramina, a histamina e outras, também conhecidas como monoaminas, são catabolizadas por diversas enzimas, destacando-se a monoamino-oxidase e a catecol-Ometil-transferase. A dopamina, sintetizada nos neurônios cerebrais dopaminérgicos a partir dos aminoácidos precursores tirosina ou fenilalanina, após a exocitose da vesícula sináptica e sua liberação na fenda sináptica, liga-se aos vários subtipos de receptor pré e pós-sináptico. Após isso, pari não se acumular progressivamente na fenda sináptica e impedir por saturação a neurotransmissão, ou é recaptada pelo neurônio pré-sináptico, através de um mecanismo ativo de membrana, ou sofre inativação enzimática. O mecanismo de recaptação pode ser influenciado, bloqueado por determinadas substâncias, de modo a que o neurotransmissor permaneça por mais tempo na fenda sináptica e possa exercer por mais tempo sua açāo nos receptores. Este é o mecanismo de ação de vários medicamentos antidepressivos clássicos, os tricíclicos, como a imipramina, a clomipramina, a amitriptilina, a nortriptilina e outros. Certas substâncias são mais ativas ao bloquear a recaptação da noradrenalina, outras da serotonina, outras ainda, da dopamina. Daí deriva sua utilidade como antidepressivos, mas também como medicamentos coadjuvantes no tratamento da doença de Parkinson, no qual estes neurotransmissores, especialmente a dopamina, se encontram acentuadamente diminuídos. Uma vez reconduzido para o interior do citoplasma do neurônio pré-sináptico, o neurotransmissor poderá ser novamente armazenado em vesículas sinápticas e reutilizado em etapas posteriores. Se não for protegido pela membrana vesicular e permanecer no citoplasma, será fatalmente metabolizado por sistemas enzimáticos intraneuronais. 
No caso das monoaminas, a enzima intracitoplasmática que inicialmente atuará é a monoamino-oxidase (MAO). A serotonina tem metabolização exclusivamente intraneuronal, pela MAO. No caso da dopamina, a metabolização intraneuronal inicia-se pela ação da MAO; a seguir, o novo produto sofre a ação da aldeídodesidrogenase, com a formação do metabólito DOPAC, o qual, finalmente sofre a ação da enzima catecol-O-metiltransferase (COMT), transformando-se no ácido homovanílico (HVA), que se constitui no metabólito final do metabolismo da dopamina. Não sendo recaptada para o interior do neurônio pré-sináptico, a dopamina sofre a ação da COMT, situada no compartimento extraneuronal na fenda sináptica, transformando-se no metabólito metoxitiramina, o qual, por sua vez é metabolizado pela MAO extraneuronal, transformando-se num produto que também acaba se transformando no HVA, após a ação da aldeílo-desidrogenase. Como vemos, a MAO e a COMT se alternam em sua ação no metabolismo da dopamina, seja dentro ou fora do neurônio, dando origem no final ao $\mathrm{HVA}^{27}$.

O parkinsonismo é a expressāo de umal deficiência na neurotransmissão dopaminérgica. No tratamento da doença de Parkinson é possível atuar nestes mecinismos enzimáticos envolvidos no metabolismo dal dopamina. A pedra angular do tratamento continua sendo a utilização de levodopa, que em última instância, promoverá uma restauração de parte da neurotransmissão mediada pela dopamina. Com a ingestão da levodopa por via oral, a maior parte será metabolizada no sangue periférico pelo mecanismo da descarboxilação, com menos de $1 \%$ da levodopa atingindo o cérebro. Ao se adicionar um inibidor periférico da descarboxilase, como a benserazida e a carbidopa, os níveis de levodopa cerebral aumentam $\mathrm{cm} 14$ vezes. Com a inibição da descarboxilase parte do metabolismo periférico da levodopa é desviado para a COMT que a converte em 3-O-metildopa. Esse desvio metabólico acaba por fazer a concentração plasmática de 3-O-metildopa maior que a da própria levodopa $a^{32}$.

\section{INIBIDORES DA COMT}

A grande concentração plasmática de 3-O-metildopa fez gerar especulaçōes sobre o seu papel no tratamento da doença de Parkinson. Inicialmente, suspeitou-se que a 3-O-metildopa tivesse um efeito antiparkinsoniano mas, depois, devido a sua mais alta concentração nos pacientes com flutuaçōes motoras, passou-se a admitirse como tendo um papel na fisiopatologia das complicações do tratamento.
Seja qual for o papel da 3-O-metildopa na doença de Parkinson, a inibição da COMT por si só levarial al um aumento na biodisponibilidade periférical da levodopa ao diminuir o seu catabolismo. O bloqueio central da COMT poderia também aumentar o efeito da levodopa ao bloquear o catabolismo da dopaminia no estriado ${ }^{28}$.

O primeiro inibidor da COMT testado foi o $\mathrm{N}$-butilgalato que demonstrou ser tóxico e foi abandonado dos testes clínicos. Novos inibidores da COMT mais potentes e seletivos e sem toxicidade têm sido testados recentemente. $O$ nitecapone, que não cruza a barreira hematoencefálica, demonstrou induzir potencialização do efeito da levodopa em animais de experimentação. O tolcapone, um inibidor da COMT em vias de lançamento comercial, tem um efeito predominantemente periférico, demonstrou que quando utilizado em conjunto com a levodopa aumenta os períodos "on" e pode diminuir a dose total da levodopa dos parkinsonianos com poucos efeitos colaterais ${ }^{32}$. Outro inibidor da COMT que poderá vir a ser comercializaddr no futuro, cujos estudos experimentais já vêm de longa data, é o entacapone.

\section{INIBIDORES DA MAO}

A MAO é uma enzima que catalisa uma reação de deaminação oxidativa, ou seja, ela elimina o radical amina, introduzindo em sell lugar o oxigênio, cujos substratos são uma série de monoaminas, como a feniletilamina, a dopamina, a noradrenalina, a serotonina, a histaminat e outras ${ }^{13}$. Localiza-se na membrana externa das mitocondrias. Os estudos procurando inibidores especíticos da MAO permitiram determinar que existem dois lipos: a MAO-A que é inibida seletivamente e de maneira irreversível pela clorgilina, e a MAO-B, cuja inibição seletiva se faz pelo L-deprenil (selegilina), de maneira irreversível, e pela lazabemida, de maneira reversível ${ }^{13}$. Os substratos específicos sob os quais elas agem sĩo a serotonina $\mathrm{e}$ a noradrenalina, para a MAO-A e a dopamina e a feniletilamina, para a MAO-B. Os genes para i MAOA e para a MAO-B estão localizados na mesma região do braço curto do cromossomal $X^{15}$. A localização celular das duas enzimals é diferente. No corpo humano como um todo, a MAO-A predomina no fígado e no sistema gastrointestinal, enquanto a MAO-B predomina nas plaquetas sanguíneas e no sistema nervoso central. Quanto à localização neuronal há também umal diferenciação, pois a MAO-A é encontrada 
preferencialmente nolocus ceruleus e no núcleo dorsal do nervo vago e a MAO-B, nas células do núcleo dorsal da rafe. $O$ interessante é que embora o substrato mais efetivo da MAO-A seja a serotonina, o núcleo dorsal da rafe, onde se localizam neurônios serotonérgicos, é sede de imunorreatividade para a MAO-B e não para a MAO-A ${ }^{14}$.

A introdução dos inibidores de MAO na terapêutica humana deu-se de maneira casual, quando, na década dos cinqüenta, tentava-se tratar pacientes tuberculosos com a iproniazida, uma droga semelhante à isoniazida. Os pacientes ficavam eufóricos, de humor elevado, de maneira diferente do que ocorria com a isoniazida. Descobriu-se que a iproniazida tinha uma ação bloqueadora da MAO. Subseqüentemente, outros derivados com ação deste tipo passaram a ser utilizados como medicamentos antidepressivos" . Os inibidores da MAO-A (IMAO-A) produzem uma inativação desta enzima nos intestinos e fígado, de modo que, ao se ingerir alimentos que contenham tiramina, e esta não sendo neutralizada pela MAO-A, podem ocorrer reações adrenérgicas violentas, com hipertensão arterial, taquicardia, cefaléia, vômitos, etc. A tiramina libera noradrenalina nas terminações nervosas simpáticas. Esta reação passou a ser conhecida como a "reação do queijo" (cheese effect). Pacientes em tratamento com estas drogas antidepressivas devem se abster de comer alimentos ou bebidas ricos em tiramina, como os queijos, chocolate, vinhos, etc. Os IMAO-B, por sua vez, não agindo na MAO-A intestinal e hepática, não produzem esta reaçĩo com os alimentos.

Birkmeyer e cols., em $1975^{\circ}$, tratando pacientes parkinsonianos com L-dopa e selegilina puderam demonstrar um aumento do efeito da L-dopa e diminuição das flutuações clínicas. Este efeito foi também demonstrado em outros ensaios clínicos, podendo produzir efeitos colaterais dopaminérgicos, como alucinações, discinesias ou confusão mental, além de efeitos de tipo anfetamínico, como insônia e irritabilidade, devido à produção de anfetaminas como resultante do catabolismo da selegilina no corpo humano ${ }^{5.20}$.

A ocorrência de parkinsonismo desencadeado pelo uso inapropriado de derivados de meperidina contaminados pelo MPTP, no início da década de 80 , trouxe um grande interesse no estudo da neurotoxicidade deste produto. A utilização de MPTP experimentalmente em alguns animais, como o camundongo e em primatas, permitiu a criaçāo de modelos experimentais de doença de Parkinson mais aproximados à doença humana que os modelos mais antigos e tradicionais. As lesões são muito semelhantes, à exceção de que não ocorrem corpúsculos de Lewy. Na realidade, o MPTP näo é verdadeiramente a neurotoxina, mas uma protoxina, pois necessita ser transformada no composto MPP+, em ordem de se tornar tóxico ao neurônio. Estal transformação se faz por uma reação de oxidação, catalisada pela MAO-B ${ }^{8}$. Este novo composto é captado pelo terminal do neurônio dopaminérgico e nas mitocôndrias exerce uma ação no mecanismo de fosforilação oxidativa, através de um bloqueio no complexo I da cadeia respiratória mitocondrial, com formação de radicais livres, levando à morte neuronal ${ }^{21}$. Este fato não ocorre, entretanto, em todas as espécies animais, sendo que algumas, como os ratos, têm uma proteçāo contra esta ação, não desenvolvendo lesĩes quando tratadas com o MPTP. No camundongo, nos primatas e no homem este mecanismo lesional ocorre. A transformação do MPTP em MPP+ pode ser bloqueada quando, previamente à alministração da protoxina, trata-se os animais com um bloqueador da MAO-B, como a pargilina ou a selegilina ${ }^{9.16}$. As células vivas, assim como os neurônios, por vários mecanismos metabólicos estāo constantemente produzindo radicais livres, como os radicais hidroxila, que são notoriamente tóxicos para as células. Entretanto, contamos com mecanismos endógenos de neutralização destes radicais livres, com sistemas enzimáticos como os da glutatione peroxidase, superóxido dismutase, catalase, etc. Um desequilíbrio entre a formação dos radicais livres e sua neutralização, provocando o que se convencionou chamar de estresse oxidativo, pode ser a causa da morte celular, por acúmulo dos radicais. A dopamina pode ser metabolizada pela MAO-B, com a consecjuente formação de peróxido de hidrogênio. Em condições normais o peróxido é neutralizado pela glutatione peroxidase, mas em condições em que este mecanismo seja deficiente, o mesmo pode formar radicais hidroxila combinando-se com íons de ferro. Este mecanismo de citotoxicidade, a partir de um aumento do turnover da dopamina, durante o tratamento com a levodopa na doença de Parkinson, por exemplo, pode ser suposto. Junto com a formação de radicais livres, a ação de substâncias excitotóxicas, como o óxido nítrico e sual participação no mecanismo de excitotoxicidade dos neurônios glutamatérgicos, também tem sido apontada como um cofator no mecanismo de agressão neuronal a ser combatido ${ }^{22}$. Ao lado de outras teorias, como o papel do envelhecimento, de fatores genéticos, a contaminaçio, ambiental por determinados elementos ainda nio discriminados, levando a um aumento do estresse oxidativo no cérebro e conseqüentemente a uma perda neuronal na substância negra com a produção da doença de Parkinson, tem sido levantada insistentemente. A 
possibilidade de uma prevenção de lesões cerebrais pelo MPTP, presumivelmente pelo bloqueio da MAO-B, impedindo a formação de radicais livres que a protoxina iria gerar na substância negra, levou à suposição de que inibidores da MAO-B (IMAO-B) pudessem ser úteis na prevenção da progressão das lesões na doença de Parkinson, desde que as substâncias existentes no meio ambiente tivessem uma ação semelhante à do MPTP. Este seria um efeito neuroprotetor. Estava lançada a possibilidade de se conseguir um tratamento preventivo, com efeito de neuroproteção.

Esta hipótese de trabalho, de utilizar a selegilina (Ldeprenil), um inibidor seletivo e irreversível da MAO$B$, em um grupo de pacientes de doença de Parkinson por tempo prolongado, comparando-o a outro grupo similar utilizando placebo, no sentido de detectar um efeito de prevenir a piora da enfermidade, conduziu ao estudo DATATOP. Este estudo multicêntrico americanocanadense envolveu 800 pacientes e teve um desenho duplo cego, selegilina ou o tocoferol (um antioxidante) contra placebo, cujo endpoint era um aumento das deficiências que necessitasse a introdução da levodopa. Tratava-se, pois, de um grupo de pacientes em fase inicial da enfermidade, ainda não recebendo tratamento com drogas dopaminérgicas. Os resultados iniciais, após cerca de 2 anos de tratamento, mostraram nítida diferença a favor dos pacientes tomando a selegilina ${ }^{22}$. Outros estudos mostraram resultados semelhantes ${ }^{20.30}$, como também o realizado em vários centros franceses ${ }^{2}$. Apesar da grande euforia inicial, a continuação do estudo DATATOP veio demonstrar que, após tempo mais prolongado, as curvas entre os grupos que recebiam ou não a selegilina convergiam, eliminando a diferença inicial a favor dos que recebiam a droga ${ }^{24}$. De qualquer maneira, a melhora inicial ainda apontava para a utilidade da droga. O conceito de que seu efeito se dava através de uma neuroproteção, atenuando os fenômenos patológicos que culminavam com a morte do neurônio dopaminérgico, passou a ser colocado em suspeita. Progressivamente, vem sendo discutido que os efeitos atribuídos à selegilina se devam a uma ação sintomática sobre a enfermidade, ao invés de uma verdadeira ação neuroprotetora e muito se tem escrito sobre o que realmente a selegilina faz no cérebro ${ }^{21,31}$. A idéia de que o efeito é neuroprotetor é muito atrativa, pois sugere que outros compostos com ação de inibição da MAO-B poderiam também ser úteis nesta tarefa e, ainda, que outros caminhos metabólicos ainda não esclarecidos pudessem também se mostrar viáveis na obtenção de uma neuroproteção eficaz e mais duradoura que a demonstrada pela selegilina. Entretanto, são vários os motivos a se considerar que uma ação sintomática da droga seja a responsável pelos resultados. Inibindo a MAO-B e, com isso, diminuindo a catabolização da dopamina estriatal, este neurotransmissor aumentaria sua concentração e conseqüentemente sua ação sintomática. Após a interrupção da selegilina, a recuperação dos níveis de MAO-B poderia demorar até 30 dias. Neste período, qualquer estudo clínico de eficácia poderia ainda detectar um efeito sintomático que poderia desaparecer após este tempo ${ }^{21}$. Além disso, a droga após ser incorporada no sistema nervoso é metabolizada em metanfetamina e anfetamina, que promovem a liberaçĩo de neurotransmissores, como a noradrenalina, podendo exercer efeitos clínicos como antidepressivos e euforizantes ${ }^{21}$. O próprio Parkinson Study Group, que conduziu o estudo DATATOP, tentando buscar uma evidência de efeito neuroprotetor da droga neste grupo de pacientes, realizou exame do líquido cefalorraquiano (LCR) com determinação dos níveis de ácido homovanílico (HVA) em amostrás colhidas antes do início do tratamento (baseline), comparando-o com os resultados obtidos no LCR colhido até 8 semanas após a retirada do medicamento (após o endpoint). Se o efeito fosse neuroprotetor, seria de se esperar uma menor diminuição dos níveis de HVA no grupo que recebeu droga ativa, em comparação ao grupo placebo. Os resultados não mostraram esta diferença, apontando na direção de não haver o efeito protetor ${ }^{26}$. Recentemente, Hao et al. apresentaram dados que sugerem a existência de substância (s) neurotóxica (s) no LCR de pacientes com doença de Parkinson, capaz (es) de inibir o crescimento e as funçóes de neurônios dopaminérựicos de ratos em cultura ${ }^{12}$. Este efeito podia ser evitado adicionando-se a selegilina à cultura. O verdadeiro papel do estresse oxidativo na etiopatogenia da doença de Parkinson e a procura de novos dados nesta área continuam'. De qualquer maneira, seja por um efeito neuroprotetor, seja por efeito sintomático, o importante é que a selegilina teve o mérito de mostrar uma nova rota de tratamento à doença de Parkinson.

A impressão de que o uso da selegilina em combinação com outros fármacos antiparkinsonianos, especialmente da composição levodopa-inibidor periférico da descarboxilase dos aminoácidos aromáticos, pudesse favorecer a melhora clínica e aumento da longevidade dos pacientes com doença de Parkinson ${ }^{4}$ foi, recentemente, objeto de uma pesquisa realizada pelo grupo britânico de pesquisa da enfermidade (Parkinson's Disease Research Group of the United Kingdom $)^{17}$. Estudaram um grupo de 520 pacientes em 93 hospitais, num ensaio aberto. randomizado, de longa duração. Estes pacientes, cm fase inicial da doençà, não haviam recebido até então 
medicamentos dopaminérgicos. Foram divididos em três grupos: num deles, os pacientes receberam levodopainibidor periférico de descarboxilase; no outro, receberam esta mesma medicação acrescida de selegilina e, no terceiro, receberam apenas bromocriptina. $O$ objetivo principal era estudar seriadamente a evolução das deficiências, a frequiência e intensidade dos efeitos adversos e, especialmente, as mortes de qualquer natureza. Com um seguimento médio de 5, 6 anos, chegaram a conclusões alarmantes de que a combinação de selegilina à levodopa não conferiu benefício maior que aquele dado apenas pela levodopa e, além disso, que a mortalidade ocorrida foi significantemente maior no grupo que recebeu a selegilina. Isso provocou um efeito monumental em todo o mundo, pois uma grande parte dos especialistas da área vem, há anos, indicando o uso da droga a todos os pacientes que possam recebêla em condições de segurança, especialmente nas fases mais iniciais da enfermidade. O ceticismo sobre a ação benéfica e uma suposta mortalidade envolvida, avaliada no mesmo número do British Medical Journal (16 de dezembro de 1995) por Donald Calne ${ }^{7}$, provocou imediata reação. Uma série de cartas ao editor (letters to the editor) do BMJ foram enviadas, com críticas aos autores do trabalho ${ }^{18}$ e, desde entāo, novas críticas foram levantadas, principalmente no tocante à metodologia empregada na pesquisa. No momento, aguardam-se novos estudos enfocando o aspecto da mortalidade, para que se possa determinar a segurança da droga a longo prazo, apesar de que a maioria dos pesquisadores se apresente cética a respeito dos resultados do grupo britânico. Temos mantido a prescrição da selegilina em nossos pacientes, com a ressalva de que são informados da existência da pesquisa do grupo britânico e da polêmica que gerou.

Um outro IMAO, a lazabemida (RO19-6327), é um inibidor de ação competitiva c altamente seletivo para a MAO-B (100 vezes mais que a selegilina), de curta ação e desprovido de efeitos anfetamínicos, pois tem estrutura química diferente. Além disso, tem uma ação reversível, ao contrário da selegilina, não necessitando de síntese de novo da MAO-B após a interrupção da sua administração ${ }^{10}$. Em ensaio clínico randomizado e multicêntrico, 20! pacientes ainda não tratados receberam lazabemida por 8 semanas ${ }^{23}$. Houve uma boa tolerância ì droga e os resultados mostraram uma melhora importante nas atividades da vida diária, embora não mostrasse melhoras significativas em outros itens da escala UPDRS. Um outro estudo multicêntrico, randomizado e duplo-cego do mesmo grupo, com 1.37 pacientes que estavam em tratamento com a levodopa, foram submetidos à droga por até 8 semanas. Devido a um aumento das reações colaterais de tipo dopaminérgico, admitiu-se uma provável ação da droga, embora do ponto de vista clínico nāo foram observados sinais de melhora evidente ${ }^{25}$.

O MDL 72,974A [(E)-4-fluoro--fluoroethylene benzene butanaminel é uma droga com atividade inibitória potente, seletiva e irreversível sobre a MAO$\mathrm{B}^{36}$. Da mesma maneira que a lazabemida, não tem açĩo anfetamínica, nem potencializa o cheese effect produzido pela tiramina. Myllylï et all. ${ }^{19}$ conduziram um estudo multicêntrico, aberto, de curta duraçĩo, em pacientes parkinsonianos que recebiam levodopa e inibidor periférico de descarboxilase, mas vinham tendo deterioração de fim de dose. Trinta e dois pacientes foram selecionados e receberam concomitantemente it sua medicação habitual, 1 ou $4 \mathrm{mg}$ da drog̣a-teste ao dia, sendo avaliados pela escala UPDRS. Os resultados sugerem que o MDL 72,974A é bem tolerado e produz melhoras clínicas significativas, nas comparaçóes da escala UPDRS. Entretanto, este foi um grupo reduzido de pacientes e o tempo de duração do ensaio foi breve.

Recentemente, $\mathrm{Yu}$ et al. ${ }^{35}$ descobriram que aminas alifáticas são rapidamente deaminadas pela MAO-B e sintetizaram uma série de compostos alifáticos $\mathrm{N}$ propargylamine, alguns deles se mostrando potentes inibidores da MAO-B e mais seletivos que a selegilinal. como $\mathrm{N}$-(2-hexyl)-N-methyl-propargylamine $102-$ HxMP] e o N-methyl-N-(2-pentyl)-propargylamine lo M-2-PP]. Estes compostos são eficazes também nat proteção contra as lesões induzidas pelo MPTP e o DSP4, em animais. Estes compostos poderio vir a se constituir em um novo grupo de drogas úteis hat terapêutica da doença de Parkinson.

Em contriste com a MAO-B, presente nos compartimentos extraneuronais, a MAO-A esta presente tanto extri- como intraneuronalmente nas terminaçöes pré-sinápticas dopaminérgicas. Ainda não se sabe bem qual a contribuição da MAO-A no metabolismo estriatal humano ${ }^{3.4}$, mas admite-se que $20 \%$ dil atividade estriatial da MAO seja desempenhada pela MAO-A. A idéia de se procurar aumentar a disponibilidade de dopamina no estriado, inibindo a sua catabolização pela MAO-A, aumentando a ação da levodopa administrada al parkinsonianos, foi estudada por Sieradzan et al. "" utilizando a moclobemida. Esta droga é existente no mercado farmacêutico, com ação antidepressiva, que atge como um inibidor reversível (RIMA) e seletivo dia monoamino oxidase A. Um ensaio pequeno, contando com 12 pacientes submetidos a tratamento dopaminérgico, nos quais a selegilina foi suspensa pelo menos 2 meses antes. Receberam 450 mg ( 3 tomadas de $150 \mathrm{mg}$ ) ao dia, durante 22 dias. Foram avaliadoso 
humor, função cognitiva e desempenho motor. Os resultados mostraram que a moclobemida tem uma ligeira atividade antiparkinsoniana sintomática e é bem tolerada pelos pacientes.

Os dados aqui apresentados permitem-nos concluir que a utilidade dos inibidores enzimáticos que agem na COMT ou na MAO, sejam do tipo B ou A, no tratamento da doença de Parkinson é inegável e abre uma perspectiva, ainda não totalmente explorada, de ajuda tanto no sentido da melhora do rendimento do tratamento como na possibilidade de se encontrarem novos compostos com possibilidade de implantar uma estratégia de neuroproteção efetiva capaz de amenizar algum aspecto do processo patológico básico na doença de Parkinson.

\section{SUMMARY}

Enzymatic inhibition, neuroprotection and the treatment of Parkinson's disease.

The metabolism of dopamine in the brain, the enzymatic systems involved in the transformation of dopamine in its metabolites and the utilization of drugs affecting these enzymes in the treatment of Parkinson's disease are reviewed by the authors. The concept of neuroprotection and how could it be done by the current therapy or with the new drugs still to be introduced in the market was discussed. Selegiline, a potent MAO-B inhibitor, has largely been responsible for the current understanding of Parkinson's disease treatment and a series of new drugs, either MAO or COMT inhibitors as lazabemide, tolcapone and entacapone among others, have been studied in its symptomatic or neuroprotective actions

\section{KEYWORDS}

MAO-inhibitors, COMT inhibitors, neuroprotection. Parkinson's disease.

\section{Referências}

1. Ahiskog, J.E.; Uitti, R.J.; Low, M.D. et al. Levodopa and deprenyl treatment effects on peripheral indices of oxidant stress in Parkinson's disease. Neurology, 46: 796-801, 1996.

2. Allain, H.; Cougnard, J.; Neukirch, H.C. Selegiline in de novo parkinsonian patients: the French selegiline multicenter trial (FSMT). Acta Neurol.Scand., 136: 73-78, 1991.

3. Arnett, C.D.; Fowler, J.S.; MacGregor, R.R. et al. Turnover of brain monoamine oxidase measured in vivo by positron emission tomography using $L(11 \mathrm{C})$ deprenyl. J.Neurochem., 49: 522-527, 1987.

4. Birkmayer, W.; Knoll, J.; Riederer, P. et al. Increased life expectancy resulting from addition of I-deprenyl to Madopar treatment in Parkinson's disease: a long-term study. J.Neural Transm., 64: 113-127, 1985.

5. Birkmeyer, W.; Riederer, P.; Ambrozi, L.; et al. Implications of combined treatment with 'Madopar' and $L$-deprenyl in Parkinson's disease. Lancet, I: 439-443, 1977.

6. Birkmeyer, W.; Riederer, P.; Youdim, M.B.H.; et al. The potentiation of the anti akinetic effect after L-dopa treatment by an inhibitor of MAO-B, deprenyl. J.Neural Transm., 36: 303$326,1975$.

7. Calne, D.B. Selegiline in Parkinson's disease. No neuroprotection effect: increased mortality. Brit.Med.J., 311 1583-1584, 1995.

8. Chiba, K.; Trevor, A.; Castagnoli Jr., N. Metabolism of the neurotoxic tertiary amine, MPTP, by brain monoamine oxidase. Biochem.Byophys. Res. Comun., 120: 547-578, 1984.
9. Cohen, G.; Pasik, P.; Cohen, B. et al. Pargyline and depreny! prevent the neurotoxicity of 1-methyl-4-phenyl-1,2,3,6tetrahydropyridine (MPTP) in monkeys. Eur.J.Pharmacol., 106: 209-210, 1985.

10. DaPrada, M.; Kettler, R.; Keller, H.H. et al. R019-6327, a reversible, highly selective inhibitor of type $B$ monoamine oxidase, completely devoid of tyramine-potentiating effects: comparison with seligiline. In Progress in Catecholamine Research. Ed.by Sandler, M. Part B: central aspects. New York: Alan R.Liss, pp.359-363, 1988.

11. Graeff, F.G. Drogas Psicotrópicas e Seu Modo de Ação. Editora Universidade de São Paulo - EDUSP (São Paulo) 110 páginas. 1984.

12. Hao, R.; Ebadi, M.; Pfeiffer, R.F. Selegilina protects dopaminergic neurons in culture from toxic factor(s) present in the cerebrospinal fluid of patients with Parkinson's disease. Neuroscience Letters, 200: 77-80, 1995.

13. Kanazawa, I. Short review on monoamine oxidase and its inhibitors. Eur Neurol, 34 (suppl 3): 36-39, 1994.

14. Konradi, C.; Svoma, E.; Jellinger, K. et al. Topographic immunocytochemical mapping of monoamine oxidase-A monoamine oxidase- $B$ and tyrosine hydroxylase in human post mortem brain stem. Neuroscience, 26: 791-802, 1988.

15. Lan, N.C.; Heinzmann, C.; Gal, A. et al. Human monoamine oxidase $A$ and $B$ genes map to $X p 11.23$ and are deleted in a patient with Norrie disease. Genomics, 4: 552-559, 1989.

16. Langston, J.W.; Irwin, I.; Langston, E.B. et al. Pargyline prevents MPTP-induced parkinsonism in primates. Science 225: 1480-1482, 1984.

17. Lees, A.J. On behalf of the Parkinson's Disease Research Group of the United Kingdom- Comparison of therapeutic effects and mortality data of levodopa and levodopa combined with selegiline in patients with early, mild Parkinson's disease. Brit.Med.J., 311: 1602-1607, 1995.

18. Letters to the Editor. Effect of adduing selegiline to levodopa in early, mild Parkinson's dis"ease. Brit.Med.J., 312: 702-705, 1996.

19. Myllylä, V.V.; Sotaniemi, K.A.; Aasly, J. et al. An open multicenter study of the efficacy of MDL 72,974A, a monoamine oxidase type $B(M A O-B)$ inhibitor, in Parkinson's disease. Adv.Neurol., 60: 676-680, 1993.

20. Myllyla, V.V.; Sotaniemi, K.A.; Vuorinen, J.A. et al. Selegiline as initial treatment in de novo parkinsonian patients Neurology, 42: 339-343, 1992.

21. Olanow, C.W. MAO-B inhibitors in Parkinson's disease Adv.Neurol., 60: 666-671, 1993.

22. Parkinson Study Group. Effect of deprenyl on the progression of disability in early Parkinson's disease. N.Engl.J.Med., 321: 1364-1371, 1989.

23. Parkinson Study Group. A controlled trial of lazabemide (RO19-6327) in untreated Parkinson's disease. Ann. Neurol., 33: 350-356, 1993.

24. Parkinson Study Group. Effects of tocopherol and deprenyl on the progression of disability in early Parkinson's disease. N.Engl.J.Med., 328: 176-183, 1993.

25. Parkinson Study Group. A controlled trial of lazabemide (RO19-6327) in levodopa-treated Parkinson's disease. Arch.Neurol., 51: 342-347, 1994.

26. Parkinson Study Group. Cerebrospinal fluid homovanillic acid in the DATATOP study on Parkinson's disease. Arch. Neurol., 52: 237-245, 1995.

27. Riederer, P.; Sofic, E.; Konradi, C. et al. The role of dopamine in the control of neurobiological functions. In The Role of Brain Dopamine. Ed. by Flückiger, E.; Müller, E.E. and Thorner, M.O. Basic and Clinical Aspects of Neuroscience Vol.3. Springer Sandoz (Heidelberg), 1-17, 1989.

28. Reches A.; Fahn S. 3-O-methyldopa blocks dopa metabolism in rat striatum. Ann. Neurol., 12: 267-271, 1982. 
29. Sieradzan, K.; Channon, S.; Ramponi, C. et al. The therapeutic potential of moclobemide, a reversible selective monoamine oxidase A inhibitor in Parkinson's disease. J. Clin. Psychopharm., 15 (suppl.2): 51S-58S, 1995.

30. Tetrud, J.W.; Langston, J.W. The effect of deprenyl (selegiline) on the natural history of Parkinson's disease. Science, 245: 519-522, 1989

31. Tipton, K.F. What is it that I-deprenyl (selegiline) might do? Clin.Pharmacol.Ther., 56: 781-796, 1994.

32. Tolosa E.S.; Valldeoriola F.; Martí M.J. New and emerging strategies for improving levodopa treatment. Neurology, 44(suppl. 6): S35-S44, 1994.

33. Youdim, M.B.H.; Lavie, L. Selective MAO-A and B inhibitors, radical scavengers and nitric oxide synthase inhibitors in Parkinson's disease. Life Sci., 55: 2077-2082, 1994.

34. Youdim, M.B.H.; Riederer, P. Dopamine metabolism and neurotransmission in primate brain in relationship to monoamine oxidase A and B inhibition. J.Neural Transm., 91: $181-196,1993$.
35. Yu, P.H.; Davis, B.A.; Boulton, A.A. Aliphatic propargylamines, a new series of potent selective, irreversible nonamphetamine-like-MAO-B inhibitors. Their structures, function and pharmacological implications. In Neurochemistry in Clinical Application. Ed. By Tang, L. And Tang S. Plenum Press, New York, pp.17-23, 1995.

36. Zreika, M.; Fozard, J.R.; Dudley, M.W. et al. MDL 72.974: a potent and selective enzyme-activated irreversible inhibitor of monoamine oxydase type $B$ with potential for use in Parkinson's disease. J.Neural Transm., 1: 243-254, 1989.

\section{Endereço para correspondência:} Luiz Augusto Franco de Andrade \& Henrique Ballalai Ferraz LAFA Rua Borges Lagoa, 1231, c/44 04038-033 São Paulo (SP) 